\title{
Direct neurobehavioral comparisons within the septohippocampal system
}

\author{
SALVATORE CAPOBIANCO, JAMES M. MacDOUGALL, and SUSAN M. FOSTER \\ Eckerd College, St. Petersburg, Florida 33733
}

\begin{abstract}
Rats sustaining total fornix transections, septal lesions, or hippocampal lesions were directly compared on two exploratory measures, nose-poke and rearing, and two avoidance measures, Sidman avoidance and passive avoidance. Total fornix cuts and hippocampal lesions resulted in an increase in rearing activity, but only fornix cuts resulted in an increase in exploratory nose-poke. Rats with damage to the septum were homogeneous to control on both tasks. All experimental groups were superior to control on the Sidman task, while both septal and fornix rats were severely impaired on passive avoidance. These results indicate a need for direct within-experiment assessment of behavioral alterations that involve anatomical manipulations.
\end{abstract}

Selective transection of the fornix bundle, which interconnects the hippocampus and septum, has been reported to result in behavioral alterations quite different from those reported for gross lesions of either area. These differences are particularly evident in testing situations involving passive avoidance responding and activity measures. For example, rats with fornix transections have been reported to show no deficits when tested in a passive avoidance task (de Castro \& Hall, 1975; Ross, Grossman, \& Grossman, 1975; Van Hoesen, Wilson, MacDougall, \& Mitchell, 1972), which is in direct opposition to studies which have shown a severe deficit when animals sustaining septal or hippocampal damage are tested on this task (Altman, Brunner, \& Bayer, 1973; Fried, 1972). A similar situation exists when examining the results of studies utilizing measures of locomotor activity such as the running wheel. Restricted damage to the medial septum results in a marked decrease in wheel running behavior (Clody \& Carlton, 1969), while hippocampal lesions result in no change (Douglas, 1967) or an increase depending on the locus and extent of damage (Strong \& Jackson, 1970). However, fornix tractotomy leads to increased activity in the running wheel and other activity measures (Capobianco \& Hamilton, 1973, 1976; Ross et al., 1975).

Whether these results reflect a qualitative difference in functioning between components of the septohippocampal axis or simply a quantitative variation in system impairment is unclear, since behavioral tasks such as passive avoidance are very sensitive to procedural variations. Ideally, functional comparisons between the septum, hippocampus,

Supported by a grant from the Eckerd College Research and Publication Council. Send reprint requests to Salvatore Capobianco, Department of Psychology, O'Hara Hall, University of Scranton, Scranton, Pennsylvania 18510. and fornix should be carried out entirely within individual experiments, in order to minimize such variations. However, it appears that experiments to date have employed, at most, only two of the critical anatomical groups, thus severely restricting explanatory hypotheses concerning differences in behavioral processes. The present study was undertaken to provide direct comparisons within the septohippocampal system on a battery of behavioral tasks which would be sensitive to dysfunctions involving behavioral inhibition and arousal. These tasks consisted of two measures of exploratory activity, rearing and nosepoke, active avoidance responding on a Sidman schedule, and a passive avoidance task which has been extensively standardized in our laboratory.

\section{METHOD}

\section{Subjects}

A total of 68 male albino rats of the Sprague Dawley strain, obtained from Marland Breeding Farms and weighing approximately $300-350 \mathrm{~g}$ at the beginning of the experiment, were used as subjects. All animals were housed singly on a 16/8-h light/dark cycle, with ad-lib access to food and water. All subjects were tested in the following behavioral tasks in the same order to minimize interactions which may arise from exposing the same animals to a different postoperative behavioral sequence. This procedure has been shown to be especially sensitive to the changes and subsequent interpretation of results which follow differential brain lesions (Capobianco \& Hamilton, 1976).

\footnotetext{
Apparatus

Exploratory nose-poke. Measures were taken in a Lehigh Valley operant chamber, reduced in size by aluminum partitions to $18 \times 25 \times 33 \mathrm{~cm}$ high. Centered in the aluminum wall was a 4-cm-diam hole located $10 \mathrm{~cm}$ above the floor The hole opened into a Plexiglas tube, $9 \mathrm{~cm}$ long, which contained a photocell and light source positioned $1.2 \mathrm{~cm}$ inside the tube. The test cage was housed in a sound- and light-attenuating chamber equipped with a ventilating fan.

Rearing. A wooden box, measuring $18 \times 18 \times 39 \mathrm{~cm}$ high and painted flat black, was used to test the rearing response. The box had a grid floor and was placed in a sound- and lightattenuated, well-ventilated enclosure. A bank of five photocells,
} 
spaced $4 \mathrm{~cm}$ apart, was positioned $12 \mathrm{~cm}$ above the floor on one wall of the compartment. The photocells were adjusted such that when the animal reared on its hindlegs, the head and shoulders would break the photocell beams, resulting in a circuit closure of the attached relay sensor.

Sidman avoidance. Behavioral testing was carried out in a 54-cm-long $\times 20$-cm-high automated shuttlebox, divided in half by a black Plexiglas barrier, $9 \mathrm{~cm}$ in height. Both sides of the hinged chamber floor were constructed of $.63-\mathrm{cm}$ stainless steel rods. Two rods were placed on the top edges of the barrier to prevent perching during shock presentations, but were not electrified at any other time.

Passive avoidance. A Plexiglas test chamber, measuring $23 \mathrm{~cm}$ long $\times 30 \mathrm{~cm}$ wide $\times 19 \mathrm{~cm}$ high, was housed in a sound- and light-attenuated modified operant chamber with a ventilating fan. A glass drinking tube was inserted through a $2-\mathrm{cm}$ hole centered on the wall $7 \mathrm{~cm}$ above the stainless steel grid floor. Contact between the spout and the grid floor completed a contact relay circuit which permitted recording the number of licks and the latency. The equipment was programmed to deliver footshock to the feet of the animal via the grid floor, according to a predetermined schedule.

\section{Procedures}

Surgical and Histological. Surgery was performed under sodium pentobarbital anesthesia $(45 \mathrm{mg} / \mathrm{kg})$ administered introperitoneally. Septal lesions were produced by passing $1.5 \mathrm{~mA}$ of anodal current bilaterally for $15 \mathrm{sec}$ through a stainless steel electrode lowered to the following bregma zero coordinates: A 2.6, L 1.0, D 5.0 from the dura. Hippocampal lesions required $2.0 \mathrm{~mA}$ for $20 \mathrm{sec}$ bilaterally at the following two separate coordinate sites: A -2.4 , L 2.0, D 3.3; and A -3.0, L 5.0, D 7.5 from the dura. Total fornix-fimbria transections were effected by a tungsten wire knife technique developed by Hamilton, Worsham, and Capobianco (1973) for dense fiber bundles. The following flat brain bregma coordinates were used for transection: A 1.0, L 1.5, D 4.5, from the dura at a $30^{\circ}$ anteriorposterior angle. Control subjects underwent identical operative procedures, except that the shaft of electrode was not inserted into the brain.

Following completion of behavioral testing, the animals were sacrificed and the brains prepared for histological examination. Forty-micron frozen sections were prepared in the frontal plane for septal and hippocampal subjects, and in the saggital plane for fornix animals.

\section{Behavioral Testing}

Exploratory nose-poke. All subjects were allowed to recover for a period of 7 days before testing was initiated. Exploratory nose-pokes were measured individually for a single 30 -min test session. When placed in the test chamber, interruption of the photocell beam operated counters which recorded the total number of pokes and the total amount of time spent poking for each of three consecutive 10-min time periods.

Rearing. Following completion of nose-poke testing, each animal was tested in the rearing apparatus. The equipment was programmed to record the total number of rearing responses for four consecutive 4-min time intervals during the test session.

Sidman avoidance responding. The third task in the behavioral testing sequence consisted of active avoidance responding on a daily 21-min unsignaled avoidance Sidman schedule, which involved no response-produced exteroceptive feedback, for 12 days. All animals were allowed $5 \mathrm{~min}$ of adaptation to the grid floor at the beginning of the first test session. Subsequent sessions were preceded by 1 -min adaptation periods. The avoidance schedule consisted of a 20 -sec response-shock interval (R-S) which could be postponed by a barrier hurdle during this period. Failure to respond resulted in the presentation of a shock every $5 \mathrm{sec}(\mathrm{S}-\mathrm{S})$ until the animal jumped to the opposite side of the chamber. All shocks were programmed to occur for $.5 \mathrm{sec}$ at $2.0-\mathrm{mA}$ intensity. The total number of responses and shocks were recorded for each test session.

Passive avoidance. During this final phase of behavioral testing, all animals were reduced to $85 \%$ of their ad-lib body weight. Passive avoidance training consisted of placing a dish containing a solution of $50 \%$ sweetened condensed milk and $50 \%$ of a $10 \%$ sucrose solution into the home cage for 1 day. The next six training sessions took place in the test chamber, where the animals were allowed free access to the solution for a period of $15 \mathrm{~min}$ to establish a stable licking response. The stability criterion required the rats to complete a minimum of 1,500 licking responses in a 10-min period. On Day 7, the animals were allowed 500 free licks, after which an FR-30 punishment schedule was instituted and remained in effect for the 20-min test period. Every 30 licks resulted in the delivery of $.16-\mathrm{mA}$ shock for $.5 \mathrm{sec}$ to the animals' feet. The total number of shocks and responses were recorded for the duration of the test session.

Statistical analyses. All data were analyzed by a two-factor (repeated measures) analysis of variance (Winer, 1971). Significant comparisons (.05 level) were carried out using the Fisher test for least significant differences (l.s.d.) to yield critical values (c.v.) for group mean comparisons.

\section{RESULTS}

\section{Histological Results}

Figure 1 shows the locus and extent of the damage in compositve reconstructions. The septal lesions typically involved nearly total destruction of the area bounded anteriorly by the genu of the corpus callosum, posteriorly by the descending columns of the fornix, ventrally by the anterior commissure, and dorsally by the corpus callosum. Examination of hippocampal lesions revealed almost total destruction of the dorsal hippocampus for all rats. The overlying cortex and dorsolateral surface of the thalamus was damaged in approximately $30 \%$ of the animals. Ventral damage was extensive with unilateral instrusion into the entorhinal cortex noted in four animals. Animals sustaining complete transection of the fornix bundle were found to have ancillary damage to the corpus callosum and, occasionally, intrusion into the anterior portion of the stria medullaris. Final group sizes for the experiment involved 22 controls, 13 septal, 9 hippocampal, and 7 fornix animals.

\section{Behavioral Results}

Exploratory nose-poke. As shown in Figure 2, there was a marked difference in the total number of exploratory nose-pokes by the various groups during the test session. The analysis of variance yielded a significant Groups effect $(F=5.22$, df $=3 / 44$, $\mathrm{p}<.01)$. The c.v. revealed that Group Fx made significantly more responses than any other group throughout the session (c.v. = 6.4). Groups Sep, Hipp, and Con were homogeneous in their rates of responding. The total amount of time spent poking was also found to be significant $(F=4.19$, df $=$ $3 / 44, p<.05)$. The c.v. of 9.3 indicated that Group Con spent a significantly greater amount of time 


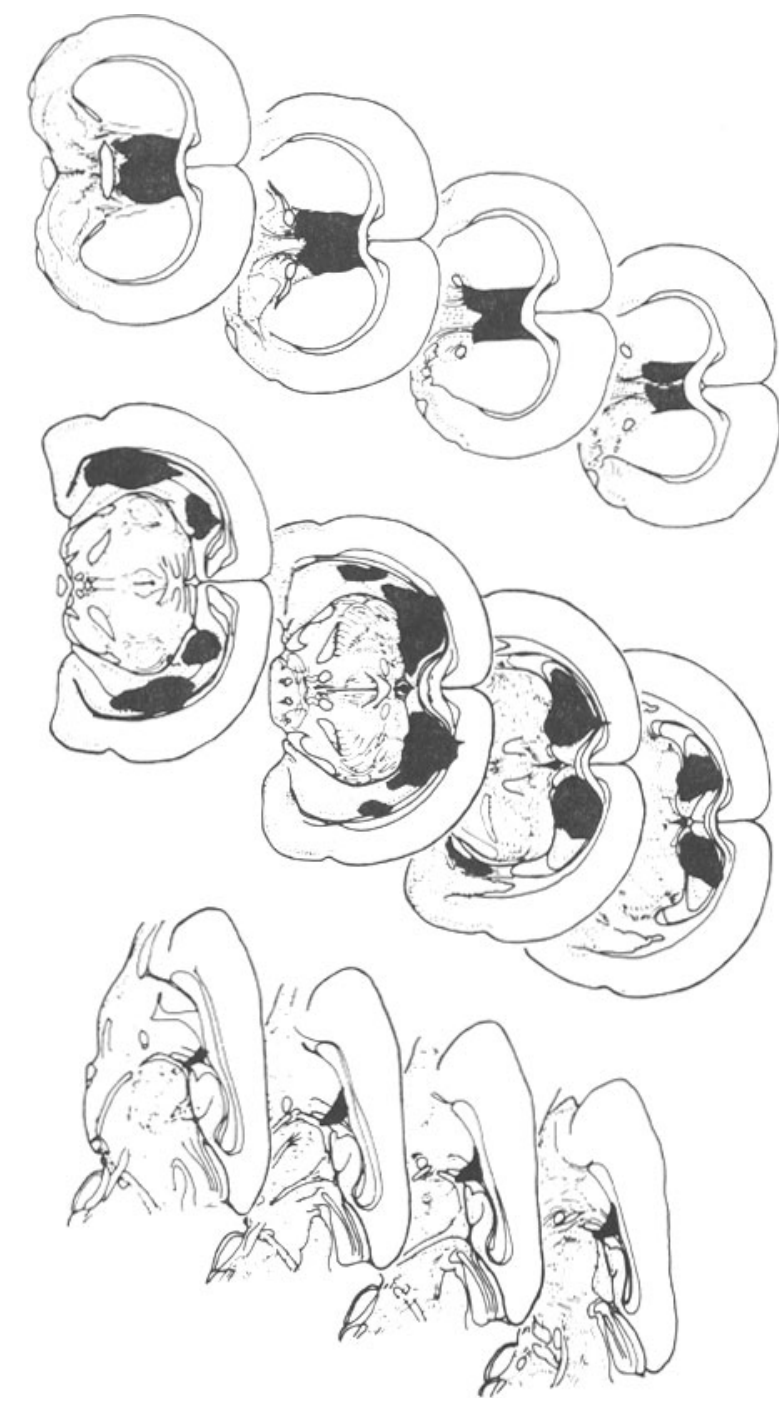

Figure 1. Composite reconstructions of locus and extent of septal and hippocampal lesions, and transections of the fornix bundle in the coronal and sagittal planes.

poking than did Groups Hipp and Sep. A similar pattern of responding was noted for Group Fx, which differed significantly from the latter groups during the first and third time intervals. The overall pattern of results indicates that selective fornix tractotomy results in a higher absolute rate of exploratory nose-poking than does gross lesions of either the hippocampus or septum, thus indicating that the fornix bundle may have an important role in mediating exploratory responding to a novel environment.

Rearing responding. The results of the rearing test, summarizing in Figure 3, show the alterations in the rearing response as a function of surgical treatment. The analysis of variance revealed a significant Groups effect $(F=4.60$, df $=3 / 44, p<.05)$, with the c.v. (7.0) indicating that Groups Fx and Hipp made significantly more rearing responses than Group Con across the entire test session. Group Sep responded in a manner similar to that of Group Con by evidencing a significantly lower response rate than Group Fx during the first two time periods in the session. It is interesting to note that hippocampal subjects reversed the large decrement in exploratory responding, as measured by the nose-poke paradigm, by evidencing a significant facilitation of exploratory activity in the rearing task. Since the fornix subjects also showed a comparable increase in activity, it is possible that this particular behavioral measure may be mediated by cell fields of a hippocampal origin, traveling through the fornix-fimbria bundle.

Sidman avoidance responding. Figure 4 shows the mean number of shocks and avoidance responses per session across the 12 days of testing. The acquisition curve for the experimental animals reached asymptotic levels of performance very rapidly; thus the number of shocks received by the brain-damaged rats was substantially fewer than the number received by the control. The analysis of variance for the total number of shocks yielded significant Lesion $(F=$ 3.82, df $=3 / 44, p<.01)$, Sessions $(F=46.6$, $\mathrm{df}=11 / 484, \mathrm{p}<.01)$, and interaction components
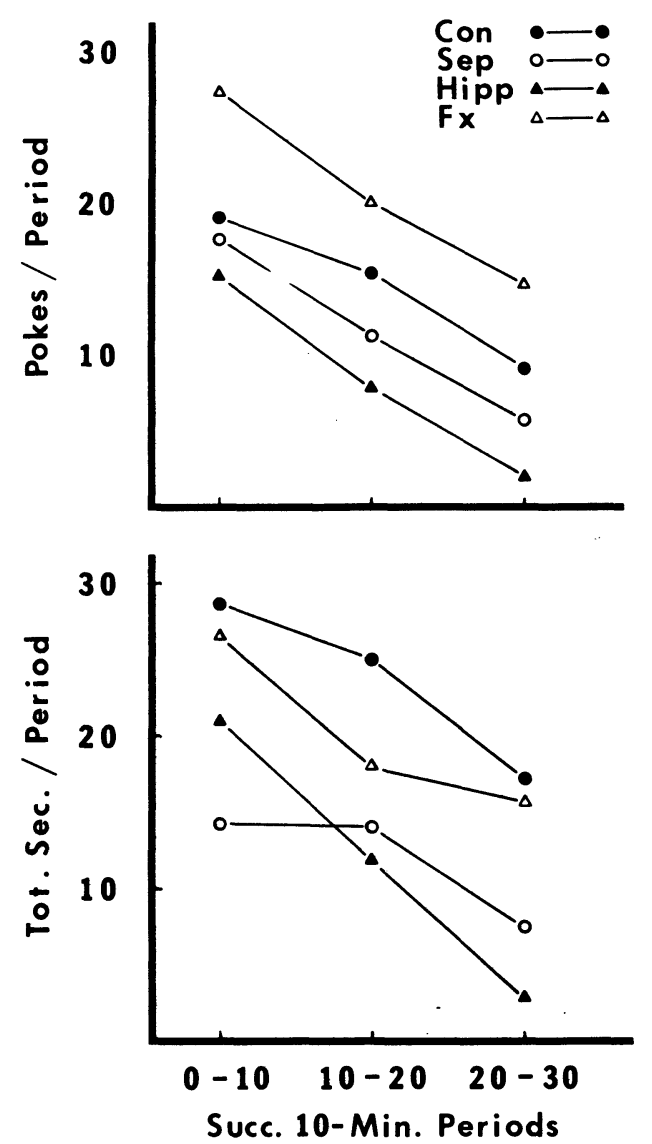

Figure 2. Total number of exploratory nose-pokes and amount of time spent poking in three successive 10 -min periods for all experimental groups. 


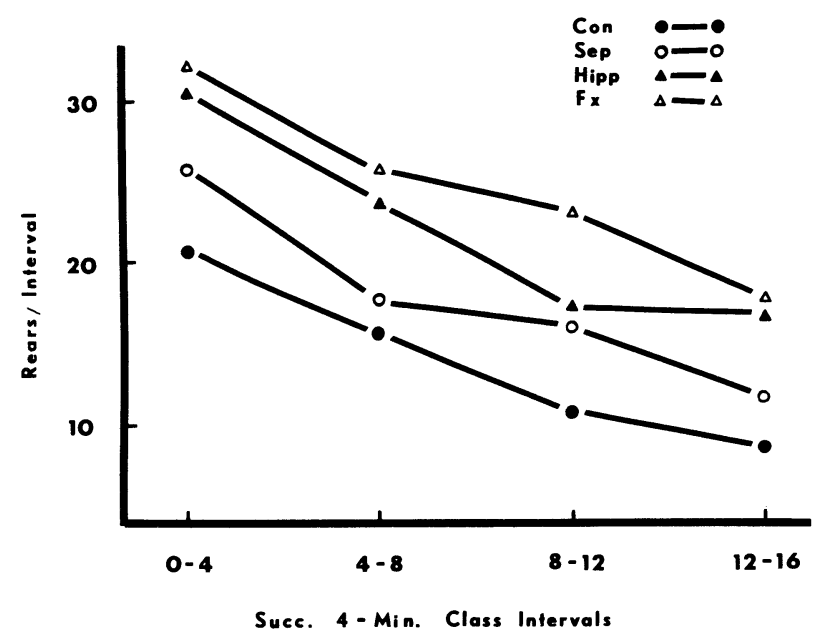

Figure 3. Total number of exploratory rearing responses made in four successive 4-min class intervals for all groups.

$(\mathrm{F}=1.72, \mathrm{df}=33 / 484, \mathrm{p}<.01)$. Within-sessions comparisons (c.v. $=19.7)$ indicated that Group Con took significantly more shocks than Groups Hipp and Sep for the entire 12 sessions, while Group Fx differed significantly from Session 6 to Session 12 . The analysis of variance for the total number of avoidance responses yielded the same general pattern of responding. The Lesion condition was found to be significant $(F=3.04$, df $=3 / 44$, $p<.05)$, as was the Sessions effect $(F=22.77$, $\mathrm{df}=11 / 484, \mathrm{p}<.01)$ and the interaction component $(\mathrm{F}=1.74, \mathrm{df}=33 / 484, \mathrm{p}<.01)$. Within-session comparisons revealed that all experimental groups made significantly more avoidance responses than did control from the 2 nd to 11 th testing session. Thus, the experimental animals acquired the avoid-

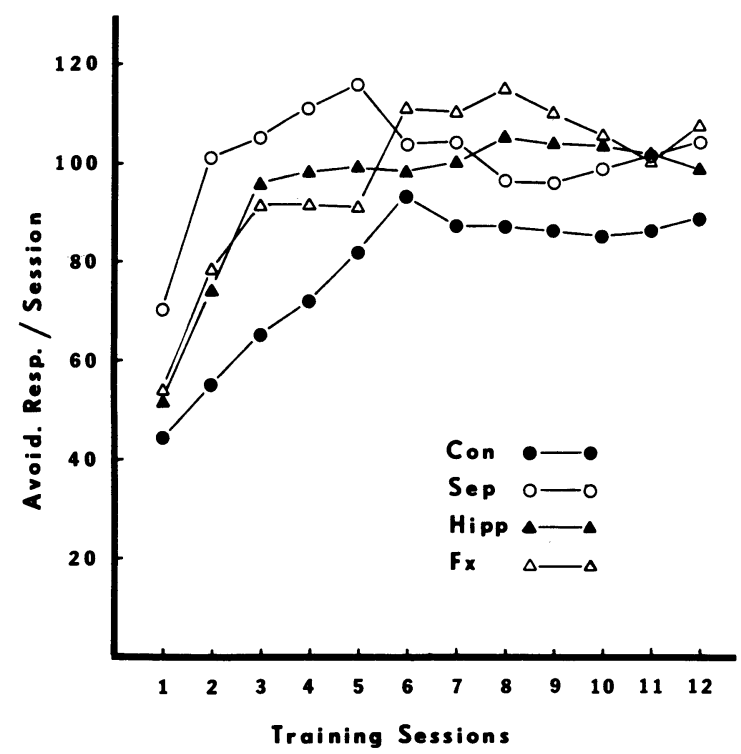

ance behavior faster, and thereby received significantly fewer shocks than control throughout the test sessions. This effect has been reliably replicated in our laboratory (MacDougall \& Capobianco, in press), and seems to be primarily due to the lengthened warm-up time required by the control animals.

Passive avoidance. The mean number of footshocks accepted during the first and second 10-min periods of the test session is shown in Table 1. Analysis of variance on the total number of shocks received during the 20-min test session yielded a significant Groups effect $(F=3.70, \mathrm{df}=3 / 45, \mathrm{p}<.05)$. The c.v. (23.1) revealed that Group Fx made significantly more punished responses, for the entire session, than Groups Sep, Hipp, and Con. The introduction of the punishment contingency was also relatively ineffective in suppressing the response rate in Group Sep, which differed significantly from Groups Hipp and Con. However, the response rate of animals sustaining damage to the septum was still significantly below that of rats sustaining transection of the fornix. Interestingly, Group Hipp did not differ from Group Con on the total number of shocks for the entire test session. However, a c.v. (8.0) performed on the first 10-min period yielded a significantly elevated response rate over Group Con (see Table 1).

\section{DISCUSSION}

The present study provides evidence for the need to carry out direct functional comparisons within the same experiment when attempting to fractionate anatomical-behavioral relationships in the septohippocampal axis, or any other functionally related central processing site. For example, the findings of

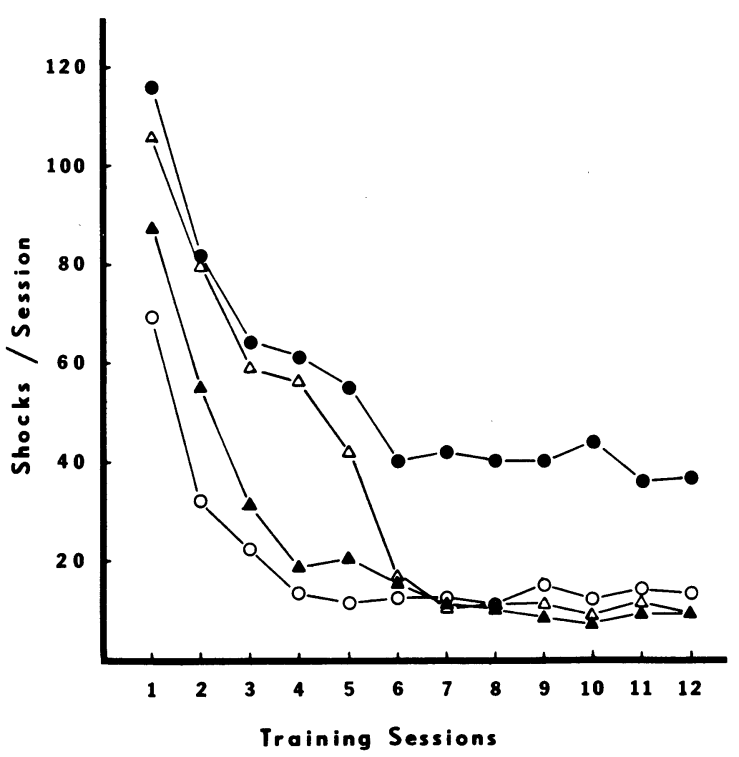

Figure 4. Mean number of avoidance responses and shocks received per session on Sidman avoidance responding for 12 days of testing by all groups. 
Table 1

Mean Number of Footshocks During the First $10 \mathrm{~min}$, the Second $10 \mathrm{~min}$, and Total for the $20 \mathrm{~min}$ Passive Avoidance Test Session

\begin{tabular}{lccc}
\hline Groups & First 10 min & Second 10 min & $\begin{array}{c}\text { Total for } \\
\text { 20 min }\end{array}$ \\
\hline Con & 9.5 & 12.3 & 21.8 \\
Sep & 39.9 & 34.8 & 74.7 \\
Hipp & 22.2 & 20.0 & 42.2 \\
Fx & 45.3 & 53.9 & 99.2 \\
\hline
\end{tabular}

Experiment 1 reveal that exploratory tendencies are differentially effected by subcomponent damage to this system. Selective fornix transection resulted in the facilitated exploratory activity in both testing situations, although hippocampectomy also increased rearing behavior. Septal lesions, on the other hand, did not increase rearing behavior from control levels, which is consistent with recent reports (Kemble \& Nagel, 1975). This pattern of responding would suggest that inhibitory hippocampal fibers traveling in the fornix-fimbria bundle may be mediating the rearing phenomena. It might be argued that the etiology of this change may be due to hippocampoentorhinal connections, since Hjorth-Simonsen (1971) has demonstrated their presence. However, studies recently completed in our laboratory demonstrate that lesions of the entorhinal cortex do not have an effect on rearing behavior (MacDougall \& Capobianco, Note 1). Consequently, hippocampal cell fields projecting through the fornix may exert control over various exploratory measures. In this regard, Myhrer (1975) has reported that selective disruption of the fimbria increases responding in the open field. Since total fornix transection would disrupt fimbrial fibers, some similarities in behavioral alterations would be expected. There is some support for this suggestion since fornix section has been reported to increase locomotor activity in the shuttlebox (Ross et al., 1975) and running wheel (Capobianco \& Hamilton, 1973).

The results of the exploratory nose-poke task showed that transection of the fornix bundle also significantly increased response levels on this measure. In opposition to the rearing task, both hippocampal and septal animals responded at a low rate throughout the test period. Previous studies have indicated that this measure is facilitated by discrete medial septal lesions (Feigley \& Hamilton, 1971). It would seem, then, that this form of exploratory behavior is rather selectively mediated by the fornixfimbria system, perhaps by fibers originating in medial septum which project to the hippocampus. Since ascending fibers from the recticular formation have been shown to project through the medial forebrain bundle and diagonal bands to septum and then to hippocampus via the fornix-fimbria system
(Guillery, 1957), it seems plausible that fornicotomy may increase arousal levels as manifested by increased locomotor activity and exploratory tendencies (Capobianco \& Hamilton, 1976; Myhrer, 1975; Ross et al., 1975).

Perhaps the most interesting result of the present study is the finding that transection of the fornix resulted in a severe deficit in passive avoidance performance. This finding is apparently in direct contradiction to other recent studies which have reported that fornicotomy has no effect on this task (de Castro \& Hall, 1975; Ross et al., 1975; Van Hoesen, MacDougall, \& Mitchell, 1969), which is confusing in view of the wide range of other inhibitory deficits. These disparate results lend themselves to at least two interpretations. First, there may be an alteration in incentive motivation by animals sustaining fornix cuts such that the increase in punished responses is due to an increased reinforcement value of the test solution. However, previous studies have failed to support a differential increase in taste sensitivity, or water intake, of rats with fornix cuts (Capobianco, 1974; Paxinos, 1975). The second, and most probable explanation, is that subtle procedural variations may account for the differences, since the passive avoidance task is very sensitive to change (see, e.g., Snyder \& Isaacson, 1965). An examination of experimental procedures revealed only one consistently obvious difference in the appetitive passive avoidance task employed by the various investigators. It was noted that the source of the aversive shock stimuli, in studies which have failed to show a deficit, was an electrified food cut or drinking tube that delivers a strong mouth shock to the animal. However, the present study utilized a scrambled footshock as the aversive stimulus and found the opposite behavioral results. This suggestion, that the shock source may be a critical determinant in assessing the results of the passive avoidance task, is strongly supported by a recent study completed by Greene and Stauff (1974). These investigators tested rats with fornix transections on a passive task for water reward which utilized scrambled footshock and found a severe impairment of performance comparable to that reported here. Although the causal explanation remains elusive, perhaps disruption of the fornix-fimbria bundle alters external spatiotemporal relationships of stimuli, such that the internal sequencing of footshock to solution is impaired. Several recent studies have confirmed the existence of a selective spatial deficit following fornicotomy in the rat (de Castro, 1974; Hirsh \& Segal, 1972; O'Keefe, Nadel, Keightley, \& Kill, 1975) and monkey (Mahut, 1972). Since there appears to be substantial support for the notion that fornix-damaged rats are unable to utilize these types of exteroceptive cues, the mouthshock vs. footshock difference in testing procedure may be a critical 
determinant in the interpretation of passive avoidance results. However, the questions concerning the precise nuclear origin of this phenomenon remain unclear, since evidently damage to either the hippocampus or septum produces similar deficits in spatial tasks (see Altman et al., 1973). It would seem that there is an obvious need to directly compare these groups on a task involving the utilization of spatiotemporal stimuli.

In contrast to the specificity evident in the previous tasks, the facilitated performance of all experimental groups, relevant to control on the Sidman avoidance task, did not provide any evidence for differential impairment of inhibitory functions. Similar results have been found in our laboratory with a variety of limbic system damage, including precommissural and postcommissural knife cuts (MacDougall \& Capobianco, in press), and appears to be primarily due to a lengthened warm-up time for control animals. Since facilitated Sidman performance is found with disparate portions of the limbic system, it would seem that a generalized increase in activity, reinforced by short IRTs, may be responsible for this effect.

It is evident from the results of the present study that there is a need for within-experiment assessment of behavioral alterations which involve complex anatomical manipulations in the central nervous system. In the present literature, such comparisons are lacking; hence, precise statements concerning functional relationships still remain somewhat tenuous. In addition, interexperimental generalizations are further complicated by subtle variations in surgical or experimental testing procedures, making precise interpretations of results difficult.

\section{REFERENCE NOTE}

1. MacDougall, J. M., \& Capobianco, S. The effects of entorhinal cortex lesions on DRL behavior in rats. In preparation.

\section{REFERENCES}

Altman, J., Brunner, R. L., \& Bayer, S. A. The hippocampus and behavioral maturation. Behavioral Biology, 1973, 8, 557-595.

CAPOBIANCO, S. The role of septal forebrain pathways in consummatory responding. Unpublished doctoral dissertation. Rutgers University, 1974.

Capobianco, S.. \& Hamilton, L. W. Increased activity following fornix transection in the female rat. Physiology and Behavior, 1973, 11, 407-410.

Capobianco, S., \& Hamiton, L. W. Effects of interruption of limbic system pathways on different measures of activity. Physiology and Behavior, 1976, 17, 65-72.

Clody, D. E., \& Carlton, P. L. Behavioral effects of lesions of the medial septum of rats. Journal of Comparative and Physiological Psychology, 1969, 67, 344-351. de Castro, J. M. A selective spatial discrimination deficit after fornicotomy in the rat. Behavioral Biology, 1974, 12, 373-382.

de CAstro, J. M., \& Hall, T. W. Fornix lesions: Effects on active and passive avoidance behavior. Physiological Psychology, 1975, 3. 201-204.

Douglas, R. J. The hippocampus and behavior. Psychological Bulletin, 1967, 67, 416-422.

Feigiey, D. A., \& Hamilton, L. W. Response to novel environment following septal lesions or cholingergic blockage in rats. Journal of Comparative and Physiological Psychology, 1971, 76, 496-504.

FrIEd, P. A. The septum and behavior: A review. Psychological Bulletin, 1972, 78, 292-310.

Green, E., \& Stauff, C. Behavioral role of hippocampal connections. Experimental Neurology, 1974, 45, 144-160.

GUILleRY, R. W. Degeneration in the hypothalamic connections of the albino rat. Journal of Anatomy, 1957, 91, 91-115.

hamilton, L. W., Worsham, E., \& Capobianco, S. A spring loaded carrier for transection of fornix and other large fiber bundles. Physiology and Behavior, 1973, 10, 157-159.

HiRsh, R., \& SEgAL, M. Complete transection of the fornix and reversal of position habit in the rat. Physiology and Behavior, $1972,8,1051-1054$.

Huorth-Simonsen, A. Hippocampal efferents to the ipsilateral entorhinal area: An experimental study in the rat. Journal of Comparative Neurology, 1971, 142, 417-438.

Kemble, E. D., \& Nagel, J. A. Persistent depression of rearing behavior in rats after extensive septal lesions. Journal of Comparative and Physiological Psychology, 1975, 89, 747-758.

MacDougall, J. M., \& Capobianco, S. Dissociation of behavioral inhibition within the fornix system. Physiology and Behavior, in press.

MaHUT, H. A selective spatial deficit in monkeys after transection of the fornix. Neuropsychologia, 1972, 10, 65-74.

MYHRER, T. Locomotor, avoidance, and maze behavior in rats with selective disruption of hippocampal output. Journal of Comparative and Physiological Psychology, 1975, 89, 759-777.

O’Keefe, J., Nadel, L., Keightley, S., \& Kill, D. Fornix lesions selectively abolish placed learning in the rat. Experimental Neurology, 1975, 48, 152-166.

Paxinos, G. The septum: Neural systems involved in eating, drinking, irritability, muricide, copulation, and activity in rats. Journal of Comparative and Physiological Psychology, 1975, 89, 1154-1168.

Ross, J. F., Grossman, L., \& Grossman, S. P. Some behavioral effects of transecting ventral or dorsal fiber connections of the septum of the rat. Journal of Comparative and Physiological Psychology, 1975, 89. 5-18.

SNYDER, R. L., \& IsaAcson, R. L. The effects of large and small bilateral hippocampal lesions in two types of passive avoidance responses. Psychological Reports, 1965, 16, 1277-1290.

STRong, P. N., JR., \& JACKson, W. J. Effects of hippocampal lesions in rats on three measures of activity. Journal of Comparative and Physiological Psychology, 1970, 70, 60-65.

Van Hoesen, G. W., MacDougall, J. M., \& Mitchell. J. C. Anatomical specificity of septal projections in active and passive avoidance behavior in rats. Journal of Comparative and Physiological Psychology, 1969, 68, 80-89.

Van Hoesen, G. W., Wilson, L. M., MacDougall, J. M., \& Mrtchell, J. C. Selective hippocampal complex deafferentation and deeferentation and avoidance behavior in rats. Physiology and Behavior, 1972, 8, 873-879.

WINER, B. J. Statistical principles in experimental design (2nd ed.). New York: McGraw-Hill, 1971.

(Received for publication September 20, 1976; revision accepted January 21, 1976.) 